\title{
CAMBIOS DE POLINIZADOR EN TIEMPO EVOLUTIVO EN PLANTAS DE PENSTEMON SCHMIDEL (SCROPHULARIACEAE)
}

\section{M.C. Castellanos}

Centro de Investigaciones sobre Desertificación (CIDE)-CSiC, Valencia, España.

Cuando se habla de biodiversidad, las plantas con flores ocupan un lugar privilegiado. En algún momento del Cretáceo tardío, la asociación con los grandes grupos de insectos polinizadores aparentemente disparó una radiación extraordinaria que fue el inicio de la impresionante diversidad en la forma y el funcionamiento de las flores que vemos hoy en día (GRIMALDI, 1999). El estudio de la evolución de las interacciones entre plantas y polinizadores es por lo tanto el estudio del origen de esa diversidad. ¿Cómo ha surgido esa enorme variación en colores, formas, tipos de recompensa floral, etc., de las angiospermas?

Existen muchas formas de aproximarse a esa pregunta y una de ellas es concentrarse en un linaje de plantas que presente variación en las flores de sus diferentes especies y, en un contexto filogenético, estudiar los mecanismos que han llevado a esa variación. Específicamente, es posible estudiar los cambios o transiciones en tiempo evolutivo de un tipo de polinizador a otro, como por ejemplo de polinización por aves a polinización por insectos, o de un tipo de insecto a otro, como de mariposa a abeja. El género norteamericano Penstemon Schmidel (Scrophulariaceae) es uno de esos grupos de plantas con flores variables y resulta ser ideal para este tipo de estudios. En este artículo explico por qué y resumo dos estudios que contribuyen a entender cómo ha evolucionado la variación (y la falta de variación) dentro de este género de plantas. Los mecanismos que han operado en Penstemon muy posiblemente son comunes a otros grupos y son responsables de parte de la diversidad de flores en general.

\section{LAS FLORES DE PENSTEMON}

Penstemon es un género exclusivamente norteamericano compuesto por unas 275 especies de hierbas o arbustos perennes (WOLFE et al., 2002). La mayor diversidad está concentrada en las montañas del oeste de Méjico y los Estados Unidos, donde tienden a florecer tarde en el verano cuando hay muchos polinizadores disponibles y hambrientos. Las flores son tubulares y producen néctar como recompensa para los visitantes. La vasta mayoría de las especies son polinizadas principalmente por abejas y algunas especies de avispas, pero hay excepciones: unas 39 especies presentan claras adaptaciones para ser polinizadas por colibríes.

Una de las razones por las cuales se trata de un grupo ideal para estudiar transiciones de un tipo de 
polinizador a otro es que dentro del género, las flores varían en forma y color y esa variación está relacionada con los diferentes polinizadores (Figura 1).

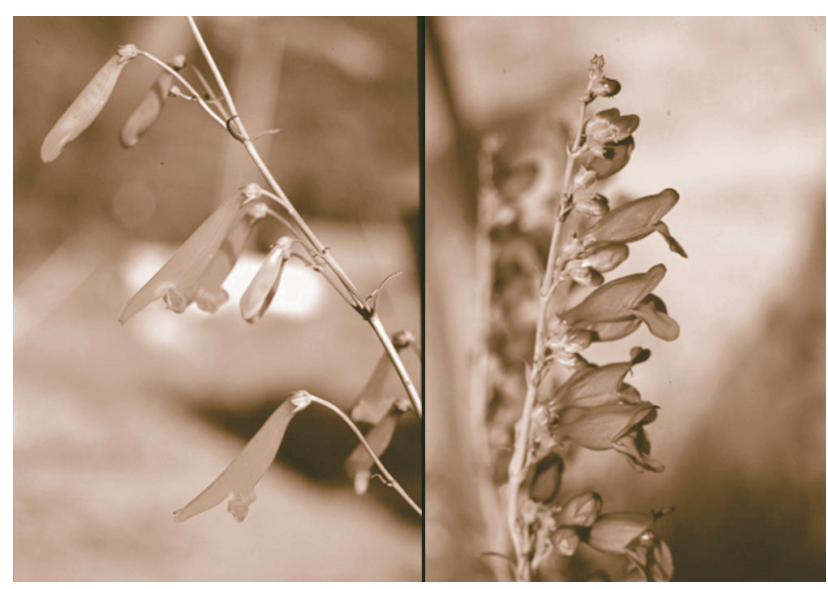

Figura 1. Inflorescencias de dos especies hermanas, Penstemon barbatus (izq., de flores rojas) y P. strictus (der., de flores moradas), de poblaciones de las montañas rocosas en Colorado (Estados Unidos). La primera es polinizada principalmente por colibries y la segunda por himenópteros.

Para demostrarlo, hicimos un estudio cuantitativo de los rasgos florales usando métodos multivariados (WILSON et al., 2004). El análisis incluyó medidas lineales de la corola, el color, el grado de inserción de los órganos reproductivos, el grado de reflexión del labio de la corola y el ángulo de inclinación de las flores de 49 especies de Penstemon. Las ordenaciones separaron las especies en dos grandes grupos a lo largo del eje principal (Figura 2): un grupo de especies con flores erguidas de tubos cortos y abiertos, de color azul o morado, con estambres y estilos insertos dentro de la corola y el labio inferior de la corola extendido formando una plataforma de aterrizaje, y otro grupo con flores colgantes rojas o naranja con corolas más largas y entubadas, órganos reproductivos que sobresalen de la corola y el labio inferior de la corola ausente o invertido. Para todas las especies teníamos además datos de los visitantes florales en poblaciones naturales. Al imponer esos datos sobre las ordenaciones encontramos una correlación fuerte entre los visitantes florales y el tipo de flor $(r=0.823$ para el vector de visitas de colibrí): las flores del primer grupo son visitadas por insectos y las del segundo grupo por colibríes (Figura 2).

En los análisis anteriores no incluimos datos del néctar producido por las flores, pero también en esto se diferencian los dos grupos. Al comparar las flores de insectos con las de colibríes encontramos que las primeras producen significativamente menos volumen de néctar, que además es más concentrado y con una mayor proporción de hexosas que sus congéneres polinizadas por colibríes (WILSON et al., 2006). En Penstemon entonces podemos hablar de dos sistemas de polinización, por abejas y por colibríes, correlacionados con dos "síndromes" florales que incluyen diferencias en el color, néctar y morfología de las flores. Es importante aclarar que estas divisiones no son absolutas. Ocasionalmente también algunos insectos visitan flores rojas en busca de polen, y con frecuencia los colibríes exploran las flores de insectos. Así mismo, los dos síndromes no explican el 100\% de la variación en los rasgos florales del género, pero sí representan el cambio macroevolutivo mas importante en las flores en Penstemon.

Esto se ve claramente cuando se grafican los dos tipos de flores en una filogenia molecular del grupo (Wilson et al., 2006). Las especies polinizadas por colibríes se encuentran repartidas a lo largo de todo el género y están en las puntas de las ramas del árbol filogenético. La interpretación más parsimoniosa indica que las transiciones han sido de polinización por insectos a polinización por colibríes y que han ocurrido de manera independiente en el grupo al menos una docena de veces, tal vez más. Esta es una situación excepcional, porque es posible entonces hacer comparaciones con control filogenético entre especies polinizadas por colibríes y sus especies hermanas adaptadas a insectos polinizadores. Esta es otra razón por la cual Penstemon es un grupo ideal para estudiar las transiciones.

\section{COMPARACIÓN DE LA EFICIENCIA DE ABEJAS Y COLIBRIES}

En muchos de los casos, esas transiciones a polinización por colibríes han sido extremas hasta el punto de excluir a las abejas. ¿Qué es lo que favorece esta especialización, que parece complicada, pues no solo implica múltiples cambios en las flores, sino 


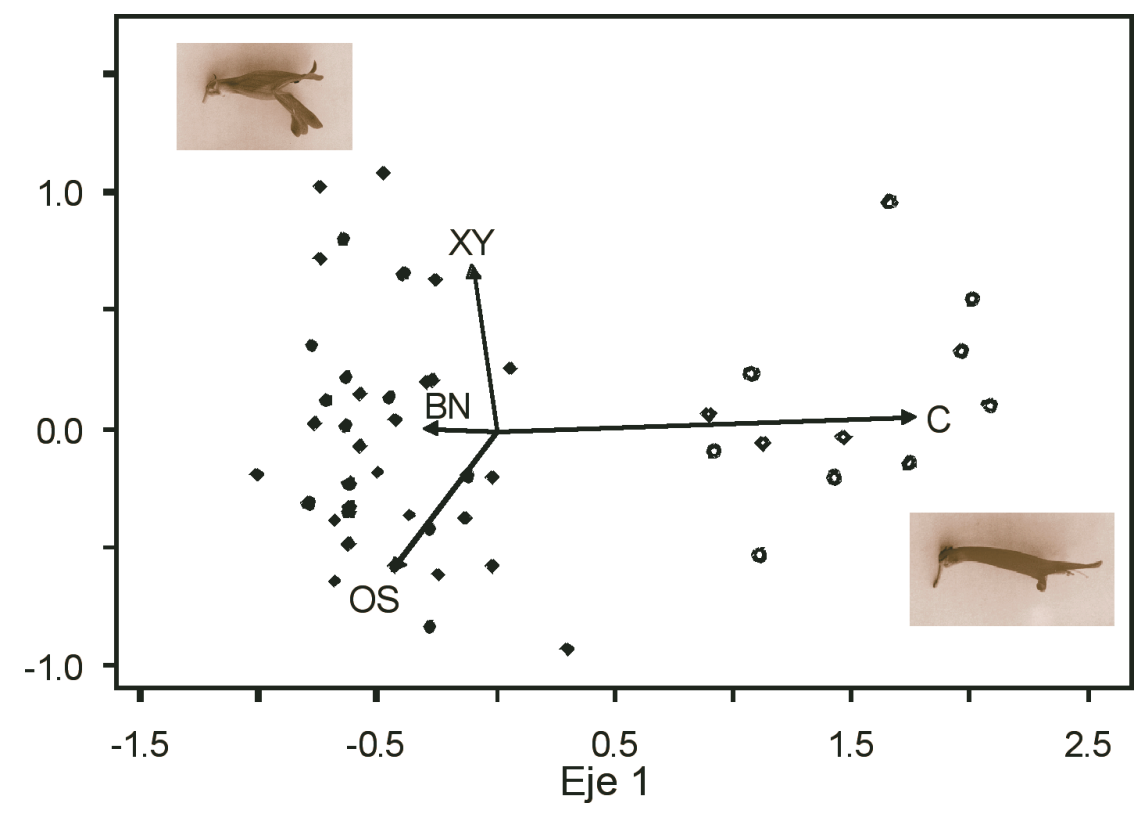

Figura 2. Ejes principales de la ordenación (tipo Non-Metric Scaling) de 49 especies de Penstemon usando 9 rasgos florales. Cada punto representa una especie; las de simbolo lleno son especies polinizadas principalmente por abejas y las de simbolo vacio son polinizadas por colibries. Los vectores son la imposición de visitas florales en censos realizados en el campo. XY: abejas del género Xylocopa, BN: abejas de Bombus consumiendo néctar, OS: abejas del género Osmia, C: colibries. El análisis separa las especies en dos grupos a lo largo del eje 1, que a su vez está muy correlacionado con la frecuencia de visitas de colibries. Las fotos son ejemplos extremos de los fenotipos florales de cada grupo. (Adaptado de Wilson et al. 2004).

que además reduce el espectro de polinizadores? Una posibilidad es que los colibríes sean polinizadores más eficientes que las abejas en estas plantas. Para poner a prueba esta hipótesis, diseñamos un estudio que comparó la eficiencia de transferencia de polen por abejorros y colibríes en dos especies hermanas de Penstemon (CASTELLANOS et al., 2003). Razonamos que es posible que los colibríes fueran más eficientes transfiriendo polen que las abejas, pues los primeros no lo consumen ni tienen adaptaciones específicas para recolectarlo, como es el caso de las abejas, que se acicalan y concentran el polen en partes específicas del cuerpo.

El estudio consistió en una serie de experimentos en el campo en los que un polinizador, un abejorro del género Bombus Latreille o un colibrí del género Selasphorus Swainson, visitaba en condiciones controladas una serie de flores de Penstemon. La primera flor visitada era donante de polen y las siguientes 15 eran exclusivamente receptoras emasculadas de polen. Con la primera visita podíamos medir el número aproxi- mado de granos de polen que removía el polinizador, contando el número de granos que permanecía en una antera por medio de un contador de partículas y comparándolo con el promedio del número de granos de las otras 3 anteras de la flor donante. Con las visitas subsiguientes medíamos el número de granos que el polinizador iba depositando en los estigmas, contando los granos bajo el microscopio después del experimento. Repetimos estos experimentos al menos 15 veces con cada tipo de polinizador en cada una de dos especies de Penstemon, P. barbatus (Cav.) Roth, ¿adaptada para polinización por colibríes y su especie hermana P. strictus Benth?, adaptada para polinización por abejas (Figuras 1 y 3). Este diseño factorial nos permitió comparar la proporción de granos removidos que ambos polinizadores depositan en otras flores (=eficiencia), en los dos tipos de flores.

La primera comparación fue entre abejorros y colibríes visitando las flores moradas de P. strictus. Los colibríes fueron sorprendentemente eficientes en transferir polen de esa especie. En promedio, los 


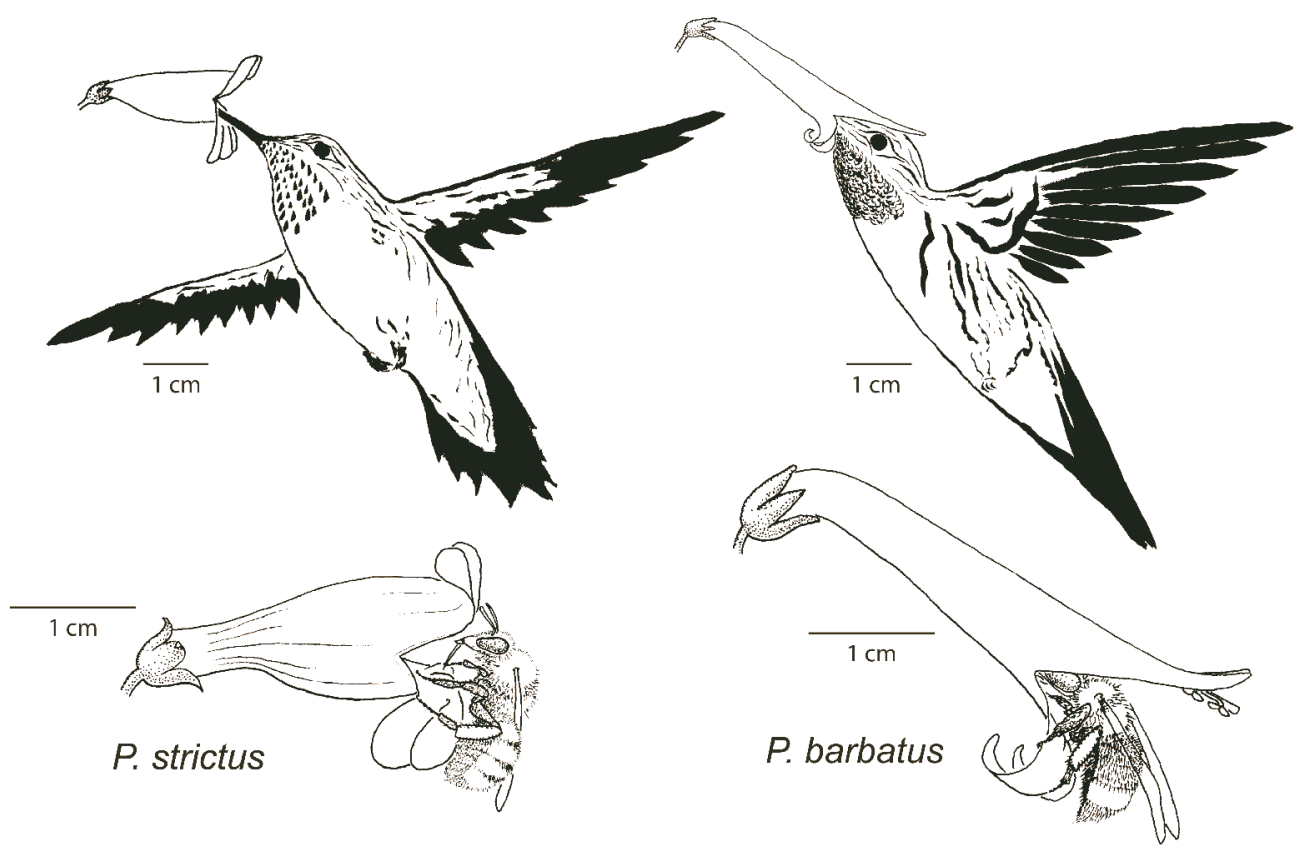

Figura 3. Colibries y abejorros visitando flores adaptadas a polinización por himenópteros y flores adaptadas a polinización por colibries. Los abejorros son obreras de Bombus flavifrons (izquierda) y Bombus bifarius (derecha) y los colibries son del género Selasphorus. (Tomado de Castellanos et al., 2004).

abejorros removieron cerca de 4.500 granos por visita y los colibríes alrededor de 3.100, una diferencia no significativa. Por otro lado, los abejorros depositaron más granos por visita que los colibríes (76 versus 32 en promedio), pero la mayoría de los granos se quedaban en las primeras flores visitadas por las abejas. Al mirar la forma de la curva de la deposición en las flores receptoras vimos que la de los abejorros tenía una caída drástica después de las primeras dos visitas, mientras que las de los colibríes prácticamente permanecían sin declive. Esto indica que más allá de 15 flores, los colibríes depositan más polen y a más flores receptoras que las abejas. En total, tanto los abejorros como los colibríes transfirieron a las primeras 15 flores más o menos el 1,6\% de los granos que habían removido, es decir, que su eficiencia era similar. En las flores rojas de $P$. barbatus, los colibríes fueron mucho más eficientes que en las flores moradas $(2,5 \%$ de los granos transferidos versus $1,6 \%$ ), lo cual es consistente con la idea de que estas las primeras están adaptadas para ser polinizadas por colibríes. Finalmente, la comparación entre abejorros y colibríes en flores rojas no es posible, simplemente porque las abejas son incapaces de visitar las flores alargadas de P. barbatus y alcanzar los nectarios.
En resumen, los colibríes demostraron ser tan buenos polinizadores como los abejorros de las flores de Penstemon adaptadas a polinización por abejas. Y además, son mucho mas eficientes en las flores rojas, que atribuimos a que la forma de tubo alargado y angosto de la flor asegura un mejor contacto de los órganos reproductivos con la cabeza y la frente del colibrí. Los cambios de las flores durante la especialización a polinización por colibríes pueden por lo tanto interpretarse como adaptaciones para optimizar el contacto físico entre flor y colibrí. Sin embargo, es posible que algunos cambios tengan la función, menos intuitiva, de excluir a polinizadores menos eficientes como las abejas. En el siguiente estudio nos preguntamos si algunos de los cambios morfológicos de las flores son también adaptaciones "anti-abeja" además de ser "pro-colibrí".

\section{LA FUNCIÓN DE LOS CAMBIOS MORFOLÓGICOS EN LAS FLORES DE COLIBRÍ}

Los fenotipos florales resultantes de la especialización a los colibríes pueden ser la consecuencia tanto de la selección para evitar visitantes florales 
no deseados, como de la selección para optimizar la interacción con las aves. En este estudio nos concentramos en cuatro rasgos florales que afectan la mecánica de la transferencia de polen: la exerción de anteras y estigma, la presencia del labio de la corola, la circunferencia del tubo floral y el ángulo de inclinación de la flor (CASTELLANOS et al., 2004). Específicamente nos preguntamos si esos rasgos cambian para excluir a las abejas del acceso al néctar o al polen, o para amoldarse mejor a la anatomía de los colibríes, o ambos.

Para detectar el efecto de la variación de estos rasgos sobre el movimiento de polen, modificamos manualmente flores de $P$. strictus cambiando uno a uno los rasgos de interés para que se asemejaran a los de su especie hermana, P. barbatus (Figura 4). En experimentos similares a los del apartado anterior, pero con flores modificadas, medimos de nuevo el número de granos de polen removido de flores donantes y el número de granos depositados en flores receptoras. Además, determinamos experimentalmente si los polinizadores se sentían o no atraídos por las flores modificadas. Los resultados fueron sorprendentes, pues sugieren que varios rasgos florales tienen función anti-abeja. Extender las anteras y los estigmas redujo el contacto con los abejorros y también el número de granos de polen depositados por ellos. También remover el labio inferior de la corola disminuyó la eficiencia de transferencia de polen por los abejorros y su atracción hacia las flores, mientras que no hubo ningún efecto sobre los colibríes. Constreñir el tubo de la corola tuvo efectos negativos sobre las abejas (tardaron mucho más tiempo en visitar una flor) y al mismo tiempo efectos positivos sobre los colibríes (aumentó la transferencia de polen). Por último, inclinar las flores también aumentó el tiempo que un abejorro necesitaba para visitarlas, pero disminuyó el la transferencia de polen por los colibríes.

De este estudio concluimos que hay potencial tanto para cambios pro-colibrí como anti-abeja. Es además muy evidente que el efecto final de estos rasgos florales sobre la atracción de polinizadores y su eficiencia no es la suma del efecto de cada uno, sino que las flores son órganos integrados en los que los rasgos cambian de manera conjunta y no independiente. Una flor más inclinada hacia abajo, por ejemplo, podría aumentar la eficiencia de trans-
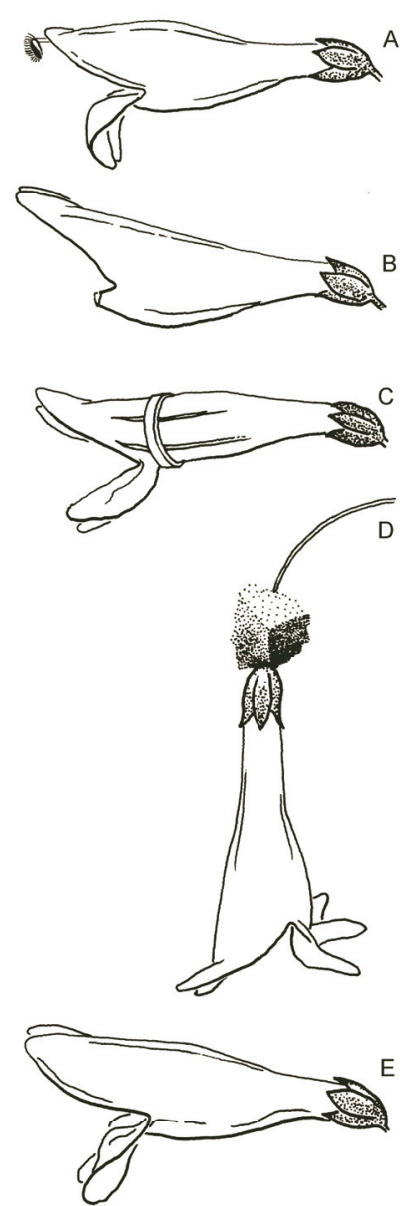

Figura 4. Modificaciones experimentales en flores frescas de P. strictus. A. Antera exerta. B. Labio inferior de la corola removido. C. Corola constreñida. D. Pedicelo felxible. E. Flor no modificada. (Tomado de Castellanos et al., 2005).

ferencia de polen por un colibrí solo si la flor tiene un tubo estrecho. A pesar de que estos experimentos ignoran el hecho de que los rasgos no cambian de manera independiente, son muy útiles para desvelar las funciones de las diferentes partes de las flores durante una transición evolutiva.

\section{UN MODELO PARA EXPLICAR LAS TRANSICIONES}

Además de los rasgos florales modificados en el experimento anterior, otras muchas características de las flores cambian en la transición a la polinización por colibríes en Penstemon y otros géneros, como resalté en la primera parte. Entre las más importantes para los polinizadores están el néctar 
y el color de la corola. Este último, es una poderosa señal para los visitantes florales, que aprenden a asociar con fuentes de néctar. Además de atraer a los colibríes, el color rojo puede ser también un rasgo anti-abeja, pues se sabe que los insectos tienen más dificultad en detectar el rojo en el fondo verde de la vegetación (CHITTKA \& WASER, 1997). Por otro lado, el néctar abundante y más diluido asociado a las flores rojas es suficientemente atractivo para los colibríes, pero no es el favorito de las abejas, que prefieren soluciones más densas (CNAANI et al., 2006). En estudios de transiciones a la polinización por colibríes en otros géneros de plantas donde éstas también han ocurrido, se ha reportado que el color, la morfología y las características del néctar tienen una base genética relativamente simple, de modo que con pocos cambios es posible el paso de un fenotipo al otro (BRADSHAW et al., 1998; BRADSHAW \& SCHEMSKE, 2003). En poblaciones naturales de Penstemon, como en P. strictus en las montañas rocosas de Colorado y en muchas otras, existe variación natural en la morfología de las flores, la tonalidad de la corola y la producción de néctar (MITCHELL \& SHAW, 1993). Los ingredientes básicos para que ocurra selección natural están presentes.

Si los cambios morfológicos y del néctar no son tan complicados y los colibríes son tan buenos polinizadores, ¿por qué entonces la gran mayoría de las especies de Penstemon son polinizadas por insectos? La respuesta muy probablemente está relacionada con la cantidad de néctar que ofrecen las flores polinizadas por insectos. El néctar es lo que en últimas determina que un colibrí realice o no visitas repetidas a una especie de planta (STILES, 1976) En condiciones normales, la oferta de néctar en una población de Penstemon no es lo suficientemente alta para atraer a los colibríes, en parte porque las flores producen pequeñas cantidades y en parte porque las abejas son visitantes frecuentes que mantienen las flores prácticamente vacías. Sin embargo, cuando una población de plantas es muy grande y el néctar se acumula en las flores, los colibríes pueden convertirse en visitantes asiduos e incluso defender territorios aunque se trate de una especie con flores de insecto. La transición a la polinización por colibríes puede entonces ocurrir cuando las condiciones ecológicas favorezcan las visitas frecuentes por los colibríes, es decir, cuando la oferta de néctar sea lo suficientemente atractiva para ellos por un tiempo prolongado. $\mathrm{Su}$ mayor eficiencia polinizando, aun en presencia de los insectos, favorecería la "invasión" de la polinización por insectos y por lo tanto la fijación de mayores volúmenes de néctar y seguidamente la aparición de los rasgos florales que optimizan la transferencia de polen por las aves y excluyen a las abejas. Si las condiciones no se cumplen o son solo transitorias, la polinización por insectos (sin exclusión de los colibríes necesariamente) sigue siendo, para una población de Penstemon, una estrategia eficiente y evolutivamente estable.

Así como ha ocurrido en Penstemon, muchos linajes de plantas se han enfrentado a situaciones similares en las que un polinizador puede convertirse en un "parásito" de polen si su eficiencia es menor que la de otro polinizador presente. Aún en presencia de varios tipos de polinizador, es posible entonces la evolución de la especialización floral. La frecuencia con la que este tipo de "dilemas" conduce a la diversificación de los fenotipos florales está aun por estudiarse.

\section{REFERENCIAS}

BRADSHAW, H. D., K. G. OTTO, B. E. FREWEN, J. K. MCKAY \& D. W. SCHEMSKE. 1998. Quantitative trait loci affecting differences in floral morphology between two species of monkeyflower (Mimulus). Genetics 149: 367-382.

BRADSHAW, H. D. \& D. W. SCHEMSKE. 2003. Allele substitution at a flower colour locus produces a pollinator shift in monkeyflowers. Nature 426: $176-178$.

CASTELlanOS, M. C., P. WILSON \& J. D. THOMSON. 2003. Pollen transfer by hummingbirds and bumblebees, and the divergence of pollination modes in Penstemon. Evolution 57: 2742-2752.

CASTELlanOS, M. C., P. WILSON \& J. D. THOMSON. 2004. 'Anti-bee' and 'pro-bird' changes during the evolution of hummingbird pollination in Penstemon flowers. Journal of Evolutionary Biology 17: 876-885. 
CHITTKA, L. \& N. M. WASER. 1997. Why red flowers are not invisible to bees. Israel Journal of Plant Sciences 45: 169-183.

CNAANI, J., J. THOMSON \& D. PAPAJ. 2006. Flower choice and learning in foraging bumblebees: effects of variation in nectar volume and concentration. Ethology 112: 278-285.

GRIMALDI, D. 1999. The co-radiations of pollinating insects and angiosperms in the Cretaceous. Annals of the Missouri Botanical Garden 86: 373-406.

MITCHELL, R. J. \& R. G. SHAW. 1993. Heritability of floral traits for the perennial wild flower Penstemon centranthifolius (Scrophulariaceae): clones and crosses. Heredity 71: 185-192.

STILES, F. G. 1976. Taste preferences, color preferences, and flower choice in hummingbirds. Condor 78: 10-26.
Wilson, P., M. C. CASTEllanOS, J. N. HOGUE, J. D. THOMSON \& W. S. ARMBRUSTER. 2004. A multivariate search for pollination syndromes among penstemons. Oikos 104: 345-361.

WILSON, P., M.C. CASTELLANOS, J.D. THOMSON \& A.D. WOLFE. 2006. Shifts between Bee- and Bird-Pollination in Penstemons. En N.M. WASER \& J. OLLERTON (eds.), Plant-Pollinator Interactions: from specialization to generalization. Chicago University Press, Chicago. Pp. 47-68.

WOLFE, A. D., S. L. DATWYLER \& C. P. RANDLE. 2002. A phylogenetic and biogeographic analysis of the Chelonae (Scrophulariaceae) based on ITS and matK sequence data. Systematic Botany 27: 138-148. 